\title{
Ethos en el liderazgo de los gestores de talento humano de las universidades y escuelas politécnicas del Distrito Metropolitano de Quito
}

Ethos in the leadership of the human talent managers of universities and polytechnic schools of the Metropolitan District of Quito

Ethos na liderança dos gestores de talentos humanos das universidades e escolas politécnicas do Distrito Metropolitano de Quito

\author{
Hilda Logaña \\ Universidad Andina Simón Bolivar, Sede Ecuador \\ hesipel.log@gmail.com \\ https://orcid.org/ 0000-0003-0838-4339
}

DOI: https://doi.org/10.32719/25506641.2020.7.10

Presentado: 8 de agosto de 2019 • Revisado: 15 y 20 de octubre de 2019

Aceptado: 5 de noviembre de 2019

Artículo de investigación

Licencia Creative Commons

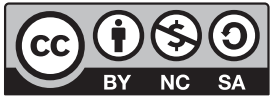




\section{Resumen}

El ethos en el liderazgo es el fundamento ontológico (existente-real-filosófico) de una profesión. Es esencial porque dictamina la disposición del gestor al ejercer las funciones que realiza debido a su profesión, y porque determina cómo lograr la plenitud profesional. El ethos no puede ser tratado de forma individual, sino como resultado de una estructura social, política, económica y hasta cultural determinada. La referencia que se hace del ethos es dinámica, porque cambia con el tiempo, se modifica y resulta ser parte de la trayectoria tanto personal como social. Entre los factores de índole personal que afectan al ethos se observan la edad, los ingresos, el tipo de educación, la estabilidad laboral y los aspectos de salud; los factores de índole social son los sistemas tanto públicos como privados (Sancén 2012).

Se realizó un estudio sobre la fundamentación teórica de todos los elementos, los conceptos, el ser moral-profesional, la estructura motivacional, el contexto de la ética, el liderazgo, y la formación del gestor de talento humano. Se contextualiza en el análisis de la universidad ecuatoriana y sudamericana, en las reformas que se han dado en ellas, y en sus estrategias de competitividad.

Palabras clave: Ethos, ética, liderazgo, gestor, recursos, humanos, valores, universidades.

JEL: J2 Reparto del tiempo; comportamiento en el trabajo; determinación y creación de empleo; capital humano.

\section{Abstract}

Ethos in leadership is the ontological foundation (existing-real-philosophical) of a profession and dictates, marks, guides the disposition of the Manager when exercising the functions that by his profession performs, as well as determines how to achieve professional fulfilment. Ethos cannot be treated individually but as a result of: a particular social structure, politics, economics and even culture; Ethos' reference is dynamic because it is changeable over time, modifiable and turns out to be part of both personal and social trajectory. Among the personal factors that affect Ethos are: age, income, type of education, job stability, health aspects and social factors including public and private systems. This study shows from the bibliography a theoretical foundation of all the elements, concepts, the moral-professional being, the motivational structure, the context of ethics, leadership, and the formation of the Human Talent Manager. The analysis of the Ecuadorian and South American university is contextualized, within the framework of the reforms that have taken place in the universities and the competitiveness strategies among them.

Keywords: Ethos, ethics, leadership, manager, resources, human, values, universities.

JEL: J2 Time distribution; work behavior; employment determination and creation; human capital.

\section{Resumo}

O Ethos na é o fundamento ontológico (existente-real-filosófico) de uma profissão e determina, marca, guia a disposição do Gestor ao exercer funções que realiza por sua pro- 
fissão, e também determina como alcançar a plenitude profissional. O Ethos não pode ser tratado de forma individual, mas como resultado de uma estrutura social determinada, da política, da economia e até da cultura; a referência que se faz do Ethos é dinâmica porque é mutante com o tempo, modificável e resulta ser parte da trajetória tanto pessoal como social. Entre os fatores de índole pessoal que afetam o Ethos, observam-se: a idade, os rendimentos, o tipo de educação, a estabilidade no trabalho e os aspectos de saúde; e nos fatores de índole social, os sistemas públicos e privados. Este estudo mostra, desde a bibliografia, uma fundamentação teórica de todos os elementos, conceitos, o ser moral -profissional, a estrutura motivacional, o contexto da ética, a liderança e a formação do Gestor de Talento Humano. Contextualiza-se a análise da universidade equatoriana e sul -americana no marco das reformas que aconteceram nas universidades e das estratégias de competitividade entre elas.

Palavras-chave: Éthos, ética, liderança, gerente, recursos humanos, valores, universidades.

JEL: J2 Distribuição de tempo; comportamento no trabalho; determinação e criação de emprego; capital humano.

\section{Introducción}

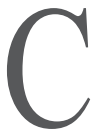

ada organización, cultura, pueblo, generación y persona posee un ethos propio. En la sociedad existen dos ethos en las relaciones de poder: los dominantes y los dominados, que, a lo largo de la historia, han sido antagónicos y a la vez complementarios, toda vez que están delimitados por la exigencia y el código moral (personal o institucional), que tiene como objetivo reglamentar, guiar y delimitar las acciones o actividades de las personas y la sociedad.

En el ámbito gerencial, los gestores de talento humano poseen como rasgo común ciertas formas de comportamiento; representan la acumulación de conocimientos, llamada también perfil duro, y aptitudes-experiencia, perfil blando. Estos elementos constituyen, en el argot de talento humano, "lo que el individuo sabe hacer (conocimiento), lo que puede hacer (aptitud, motivación, inteligencia emocional) y el poder hacerlo (ambiente-cultura, y diversas situaciones organizacionales)" (Rodríguez y Bustamante 2008, 219). El ambiente diario de trabajo permite que las competencias gerenciales se adquieran, aprendan o afinen, en vista de que el trabajo del gestor de talento humano debe manejar procesos con acciones determinadas para lograr los objetivos de la organización. 
El profesional de talento humano debe estar en constante capacitación y formación, ya que su cargo requiere la toma de decisiones y la ejecución de procedimientos. Por este motivo, se hace necesario que actualicen, aprendan o reaprendan conocimientos, técnicas y herramientas para mejorar su trabajo.

De Lella, en referencia al ethos, observa que en la academia se logra la perfección diaria del individuo en los diversos procesos y actividades que tiene a cargo, y que van ligadas a la obligación moral, al compromiso con la comunidad y a la competencia profesional. Lo resume de manera clara en la siguiente frase: "La formación constituye un proceso permanente de adquisición, estructuración y reestructuración de conductas (conocimientos, habilidades, valores) para el desempeño de una determinada función" (1999, 3).

El enfoque instrumental y funcionalista de los modelos de gestión de la organización generalmente responde a una lógica de mercado que Habermas (1973) señala como mundo objetivo, el consolidado por un orden deseado. Esta lógica consiste en alimentar el sistema capitalista, que busca maximizar el beneficio económico y acumular capital. Sin embargo, la teoría crítica pone en evidencia que la práctica gerencial no está pautada exclusivamente en esta instrumentalidad, y que existe una demanda por incluir en los desafíos gerenciales la reflexión sobre cuestiones éticas, sociales, ambientales, emocionales y humanas.

Ecuador también muestra importantes avances en el marco legal, que rescata el buen vivir como eje central para el desarrollo humano. La Constitución de la República del Ecuador, en el numeral 5 del artículo 3, consagra como deber primordial del Estado "planificar el desarrollo nacional, erradicar la pobreza, promover el desarrollo sustentable y la redistribución equitativa de los recursos y la riqueza, para acceder al buen vivir" (EC 2008).

El ethos en el liderazgo del gestor de talento humano se caracteriza y describe en todos sus aspectos, con lo que logra revelar sus comportamiento y tendencias. El presente trabajo busca conocer a este grupo social, en su conjunto de actitudes, valores y hábitos. El objetivo del gestor de talento humano es alinear la estrategia departamental con la institucional; esta acción, sumada a una dirección, un liderazgo y una comunicación efectivos, coadyuva a lograr el éxito institucional. 
El locus de la investigación es la universidad ecuatoriana, tanto la pública como la privada, considerando que desde 2015 la institucionalidad de las entidades de educación superior está atravesando continuos cambios.

El objetivo de la investigación es, principalmente, identificar la teoría y práctica de las competencias gerenciales necesarias (técnicas, decisorias y de relacionamiento) en la labor cotidiana del gestor del talento humano, a fin de reconocer el alcance de las propuestas de formación para su quehacer organizacional. Se han definido e identificado las variables del ethos gerencial como elementos necesarios de la formación para el desarrollo de competencias gerenciales.

\section{El ethos y sus componentes}

En la actualidad se ha retomado el tema del ethos para las ciencias sociales. El término puede tener distintos usos, tanto en el ámbito político como en el académico, y por eso es necesaria la comprensión teórica, epistemológica e ideológica del ethos gerencial. Con miras a analizarlo de manera empírica, se utilizó el discurso argumentativo con múltiples enfoques teóricos, desde una posición estratégica instrumental que se describirá más adelante.

El término ética tiene su nacimiento en la filosofía griega y en la retórica de Aristóteles (335 a. C.). El ethos fue referenciado en primera instancia por Homero como el sitio donde confluían tanto los hombres como los animales; en sus poemas se establece que "el modelo ethos es el individuo perteneciente a la nobleza, valiente, fuerte, justo, hábil, inteligente, rico, magnánimo, y cuya virtud principal es la de cumplir perfectamente la función social que le corresponde" (Octavi y Gomis 1987, 43). Sin embargo, para Zenón de Citio, ethos es la fuente de la vida, de la que emanan actos singulares -el hacerlos bien- (Aranguren 1958).

López Ruiz (2007) se refiere al ethos como el orden normativo personal internalizado, a partir del cual los gestores conducen su vida. Afirma también que en la actualidad, para acelerar el capitalismo creciente del siglo XXI, se hace necesario caracterizar los ethos de perfiles particulares y de las diferentes especializaciones del saber que permitan nutrir y revitalizar el espíritu de la profesión para la cual uno se está preparando y consolidando. 
Yurén (2005) se refiere al ethos gerencial como el sistema disposicional que se configura para resolver los problemas sociomorales que surgen en el campo del trabajo gerencial, que se ejerce diariamente. Romero y Yurén (2007) observan que el ethos gerencial resulta de la combinación de la ética de los gestores, la estructura motivacional y las formas de autorregulación que hacen congruentes la conducta con la moralidad construida y la ética aceptada: el ser moral al que se aspira en lo profesional.

El respeto mutuo y la tolerancia sobre la manera de pensar de las personas que intervienen el trabajo diario dan como resultado un enriquecimiento personal. La autoformación individual y la conformación, en conjunto con la gestión de grupos, permiten al gestor manejar diferentes puntos de vista, y es ahí donde se obtiene la prudencia profesional.

En esta etapa se va de la teoría a la praxis y de allí a la práctica reflexiva, ya que el día a día genera un conflicto de valores propios, grupales e institucionales para cuya resolución se hace necesario utilizar la sabiduría o experiencia previamente adquirida. La resolución de las situaciones diarias implica, según Romero y Yurén $(2007,25)$, "la equidad en base a la aplicación de la norma, la respuesta moral que se da al interlocutor, la perseverancia moral que está fundamentada en el código de conducta profesional”.

\section{La ética y su contexto}

La ética se entiende como un cuerpo de conceptos filosóficos, en los cuales se observan grupos de comportamientos morales o amorales, en los que interviene el ser interno. La ética del gestor de talento humano se complementa y se hace necesaria al momento de ejercer las responsabilidades y funciones que le son encomendadas.

La ética es fundamental en el gestor de las instituciones educativas, puesto que es el encargado de manejar contratos de personal, de resolver conflictos individuales o departamentales y de proporcionar los mecanismos y ambientes necesarios que proporcionen la motivación necesaria y, por ende, aumenten la productividad de la institución.

La ética es el arte de saber vivir con valores, con calidad humana; es saber vivir una vida que valga la pena, no centralizada solamente en los propios 
horizontes, sino también en ayudar a otros a vivir una vida plena, buscando la realización de la comunidad (Martínez 2010a). A la ética se la describe como la ciencia que estudia de una manera reflexiva la conducta humana, que se traduce en actos concretos y debe estar dispuesta a guiar el desarrollo de las personas y del colectivo social (Martínez 2010b).

Según Sonnenfeld (2012), la ética tiene un sentido que va de lo particular a lo general, de lo personal al bien común. Busca conseguir una vida en armonía y con respeto hacia el resto de las personas, es decir, la concordancia entre la ética en primera persona y la ética en tercera persona.

\section{Caso de estudio: Gestores de talento humano de las universidades y escuelas politécnicas del Distrito Metropolitano de Quito}

Esta investigación busca identificar las competencias gerenciales necesarias (técnicas, decisorias y de relacionamiento) en la labor cotidiana del gestor del talento humano, a fin de identificar el alcance de las propuestas de formación para su quehacer organizacional en las instituciones educativas universitarias.

Las instituciones educativas de categoría A y B mantienen un alto nivel académico y administrativo, siendo también reconocidas por su compromiso con las funciones sustantivas de una universidad: docencia, investigación y vinculación con la colectividad. Por este motivo, la población del estudio es considerada relevante y se constituyó de veintidós gestores y segundos al mando de los departamentos de Talento Humano de las once universidades y escuelas politécnicas del Distrito Metropolitano de Quito incluidas en las categorías mencionadas, y que se detallan a continuación. 
Tabla 1

Categorización de universidades

\begin{tabular}{|c|c|}
\hline \multicolumn{2}{|c|}{ Oferta académica de pregrado y posgrado } \\
\hline Universidad pública & Universidad privada \\
\hline Categoría A & Categoría A \\
\hline \multirow[t]{6}{*}{ Escuela Politécnica Nacional } & Universidad San Francisco de Quito \\
\hline & Categoría B \\
\hline & Pontificia Universidad Católica del Ecuador \\
\hline & Universidad Particular Internacional SEK \\
\hline & Universidad Politécnica Salesiana \\
\hline & Universidad Tecnológica Equinoccial \\
\hline \multicolumn{2}{|c|}{ Categorización de universidades con oferta académica de pregrado } \\
\hline \multicolumn{2}{|l|}{ Universidad privada } \\
\hline \multicolumn{2}{|l|}{ Categoría B } \\
\hline \multicolumn{2}{|l|}{ Universidad de los Hemisferios } \\
\hline \multicolumn{2}{|c|}{ Categorización de universidades con oferta académica de posgrado } \\
\hline Universidad pública & Universidad privada u otra denominación \\
\hline Categoría B & Categoría A \\
\hline \multirow[t]{2}{*}{ Instituto de Altos Estudios Nacionales } & Facultad Latinoamericana de Ciencias Sociales \\
\hline & Universidad Andina Simón Bolívar \\
\hline
\end{tabular}

Fuente: EC CEAACES (2015).

Para sistematizar los datos recopilados y analizarlos se utilizó el programa NVivo, lo que permitió sacar provecho de la información cualitativa desde varios enfoques analíticos. 


\section{Metodología}

Los fenómenos de la investigación se construyen a partir de realidades. De la unión de todas ellas se obtienen totalidades con una lógica propia de estructuración en la investigación.

El estudio realizado fue de carácter exploratorio, con un análisis mixto (cualitativo/cuantitativo). La metodología para la investigación parte de un eje teórico: teoría crítica, ethos corporativos y gerencial, contexto organizacional de la universidad. El instrumento de medición fue la encuesta, para verificar competencias y percepciones.

Los insumos de la investigación son los datos primarios de competencias y formación y las observaciones del comportamiento de los gestores de recursos humanos. Las dimensiones estudiadas en la investigación empírica son las de ética, decisión, aprendizaje, conocimiento, innovación organizacional y estilo de liderazgo.

Se utilizó también la técnica de entrevista a profundidad, cuya característica principal, según Olaz $(2012,7)$, es que el entrevistador cuenta con un guion tentativo de aquellas cuestiones sobre las que quiere profundizar. Suele destinarse a personas conocedoras de un tema específico, y su uso se recomienda cuando se pretende ampliar el conocimiento sobre un problema mínimamente estructurado, como es el presente caso. La ventaja de su aplicación es la flexibilidad en la estructura y en las posibles respuestas.

Una vez recabada la información, se realizó la sistematización de datos, que consistió en el análisis de contenido de cada una de las entrevistas mediante el programa NVivo.

\section{Resultados}

Se determinaron las funciones, los procesos prioritarios, los indicadores con que trabajan, las competencias y los valores que rigen la jornada de trabajo diario de los gestores de talento humano. Previo a esta determinación, se realizó un estudio sobre las normas y el funcionamiento de cada una de las instituciones educativas superiores. 


\section{Análisis de la información de las universidades estudiadas}

La información relacionada a la visión, la misión, el código de ética, los valores, el tipo de financiamiento y la dimensión de las once universidades y escuelas politécnicas con categoría A y B del Distrito Metropolitano de Quito se obtuvo de sus sitios web. A partir de un cuadro comparativo se efectuó un análisis de las similitudes y divergencias en cuanto a las características mencionadas anteriormente.

De las once universidades estudiadas, cuatro pertenecen a la categoría A y las siete restantes, a la B. Otro aspecto importante es que tres son de posgrado y ocho, de pregrado.

Figura 1

Categorización de universidades

Universidades vs. Categoría

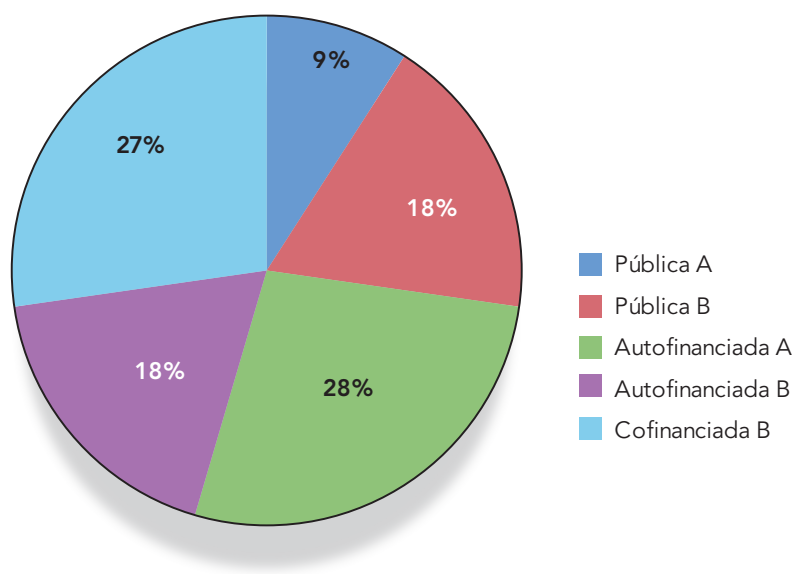

Elaboración propia.

De la información presentada en las páginas institucionales se realizó un análisis del contenido de la misión y la visión de las once instituciones. Se tomaron en cuenta similitudes y se obtuvieron los gráficos 2 y 3 . 
Ethos en el liderazgo de los gestores de talento humano de las universidades y escuelas politécnicas

Figura 2

Misión de las universidades

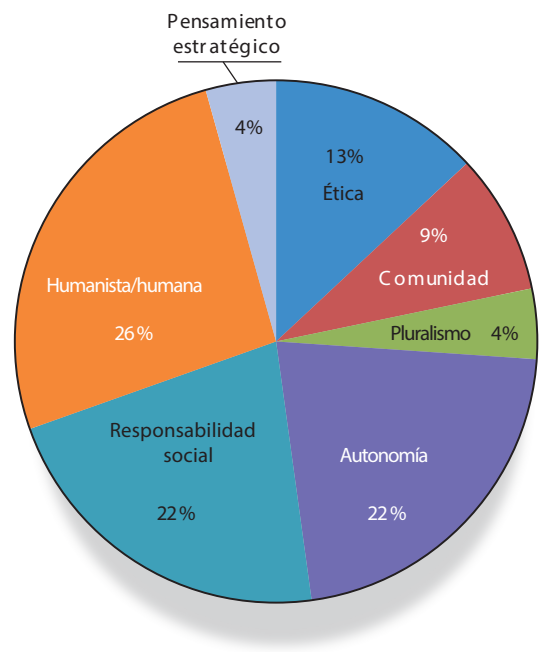

Elaboración propia.

Figura 3

Visión de las universidades

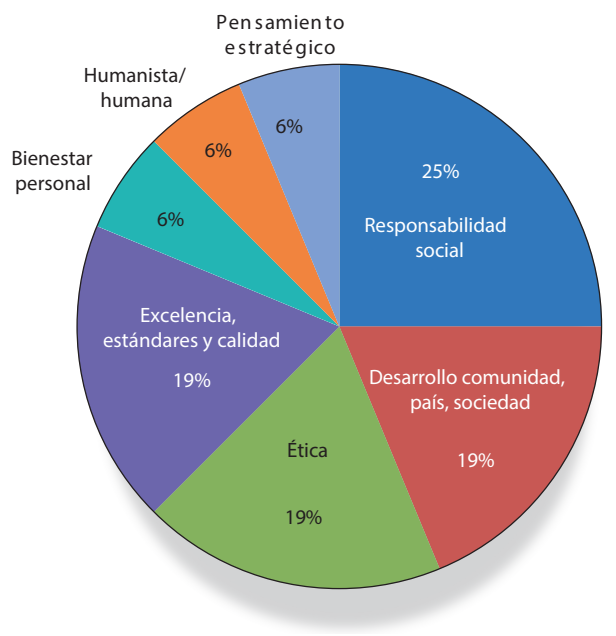

Elaboración propia. 
Figura 4

Valores de las universidades

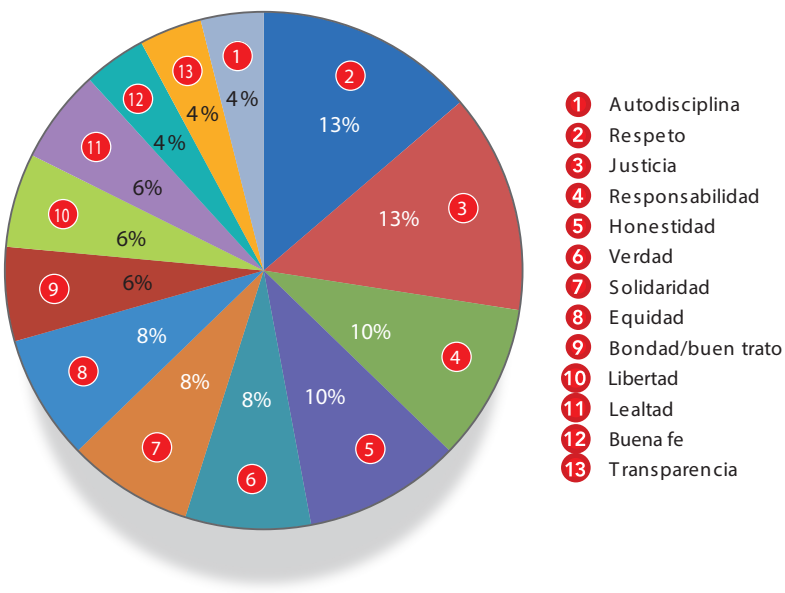

Elaboración propia.

Cabe mencionar que la innovación está presente en todos los centros universitarios del país, porque constantemente están retroalimentando información, actualizando conocimientos y realizando investigación, inversión social y vinculación con la comunidad (Randall y Jackson 1987). El Estado, a través del CEAACES, ha logrado la categorización de universidades ecuatorianas, un proceso que lleva a que estén en la búsqueda constante de la excelencia. 


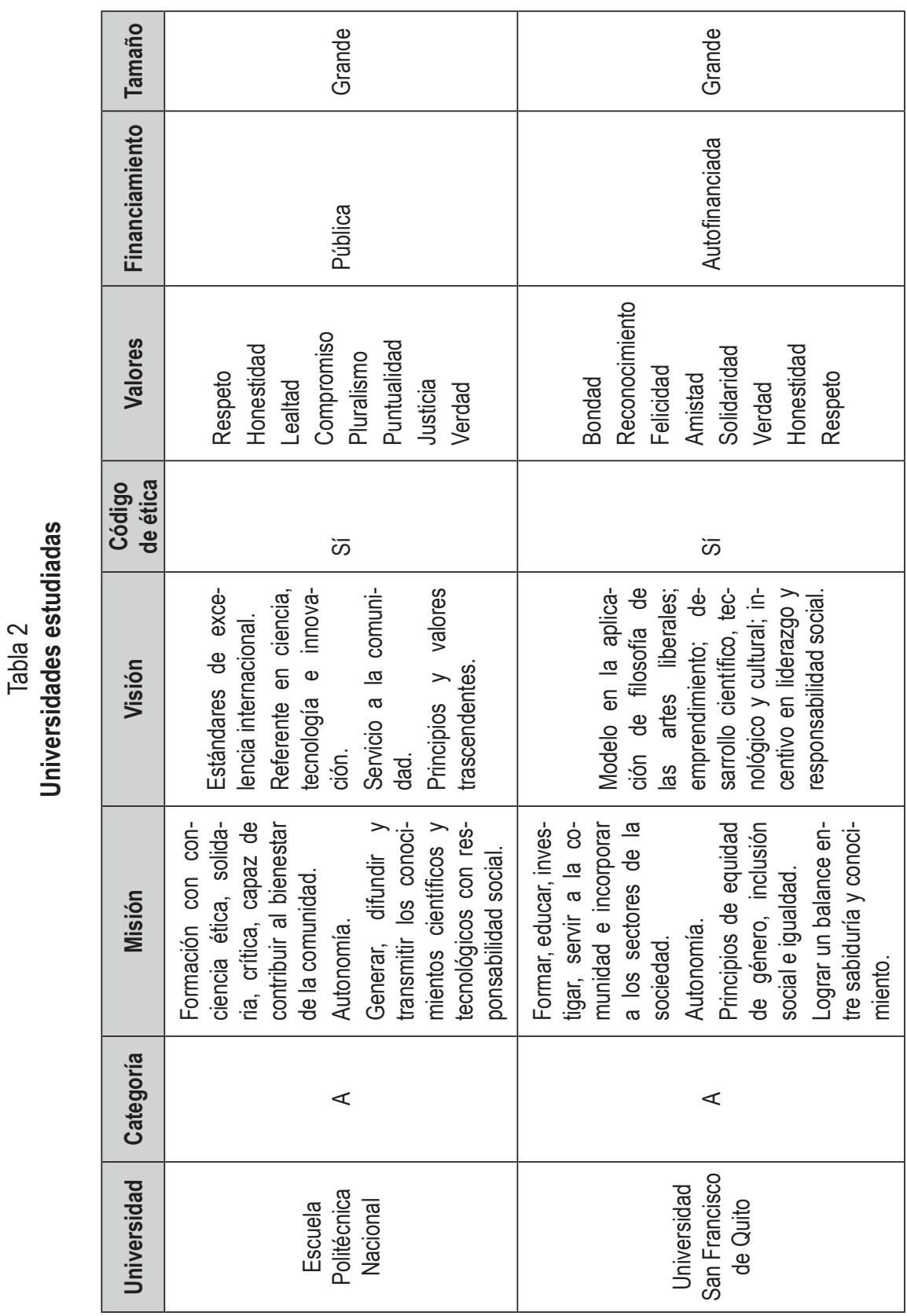




\begin{tabular}{|c|c|c|}
\hline 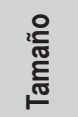 & $\begin{array}{l}\frac{10}{\circ} \\
\frac{\pi}{\pi} \\
\frac{\pi}{0}\end{array}$ & 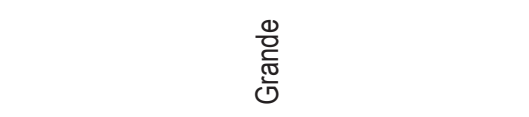 \\
\hline 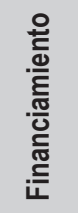 & $\begin{array}{l}\frac{\pi}{0} \\
\frac{\pi}{0} \\
\frac{0}{0} \\
\frac{\pi}{E} \\
0 \\
0 \\
0\end{array}$ & $\frac{\text { 岢 }}{\frac{0}{2}}$ \\
\hline 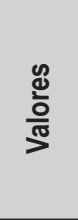 & 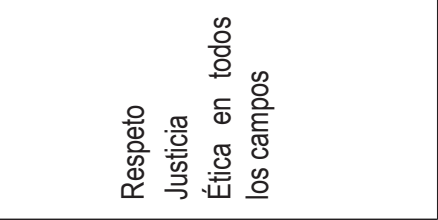 & 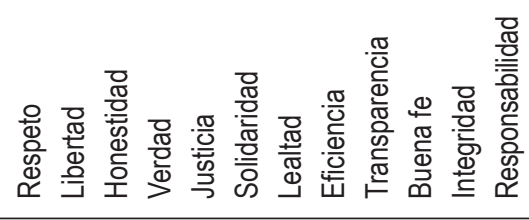 \\
\hline 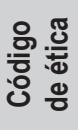 & ఉ & ๘ \\
\hline$\frac{\overline{0}}{\frac{0}{0}}$ & 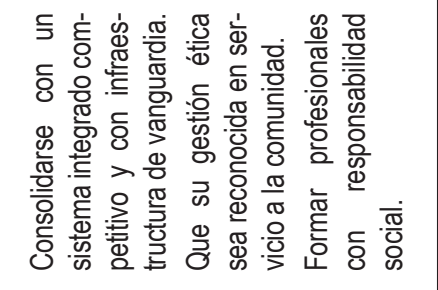 & 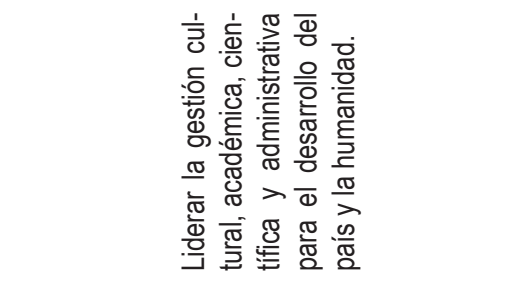 \\
\hline$\frac{\overline{0}}{\frac{0}{20}}$ & 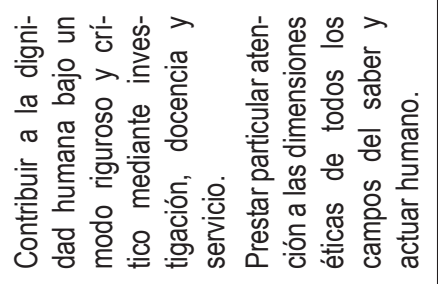 & 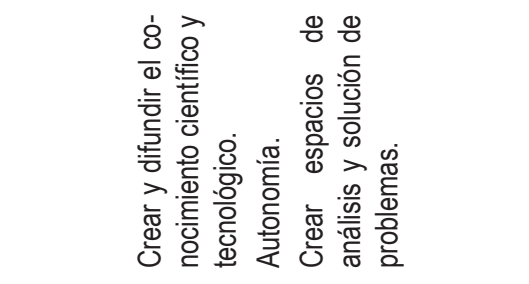 \\
\hline $\begin{array}{l}\frac{\pi}{\vdots} \\
\text { क्ष } \\
\text { సँ }\end{array}$ & $\infty$ & $\infty$ \\
\hline 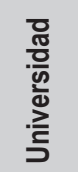 & 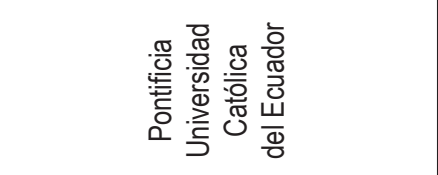 & 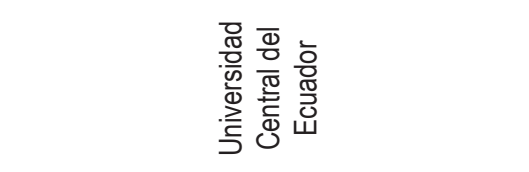 \\
\hline
\end{tabular}




\begin{tabular}{|c|c|c|}
\hline $\begin{array}{l}\frac{\mathbb{0}}{0} \\
\frac{\mathbb{\pi}}{\mathbb{N}}\end{array}$ & $\begin{array}{l}\frac{\mathbb{0}}{0} \\
\frac{\pi}{0} \\
\frac{\pi}{0}\end{array}$ & $\begin{array}{l}\frac{\mathbb{1}}{0} \\
\frac{\mathbb{\pi}}{0}\end{array}$ \\
\hline 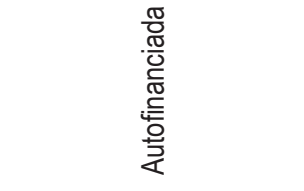 & 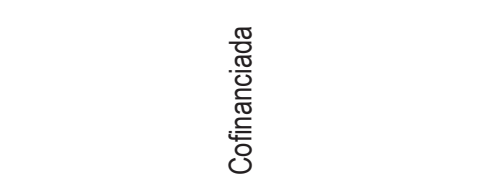 & 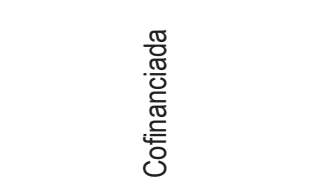 \\
\hline 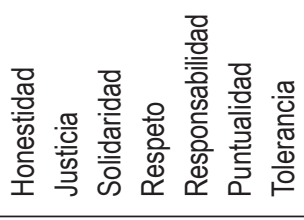 & 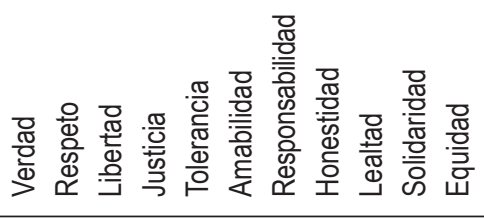 & 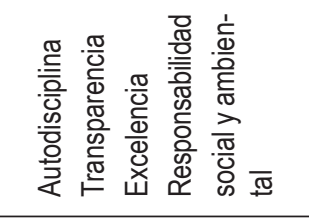 \\
\hline ఉ & is & ఉ \\
\hline 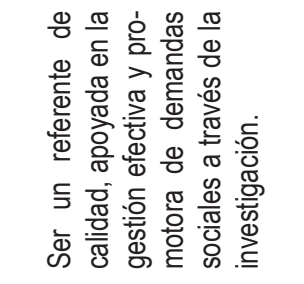 & 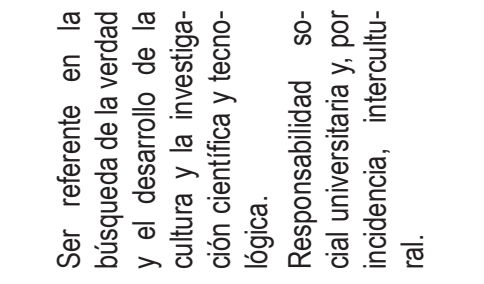 & 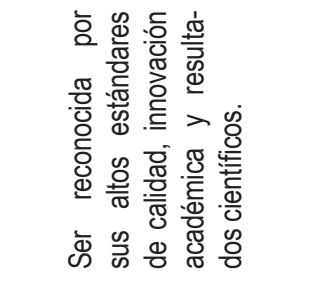 \\
\hline 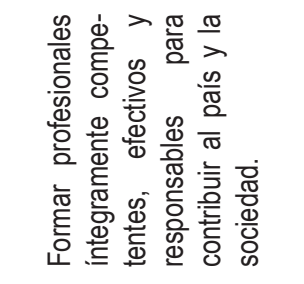 & 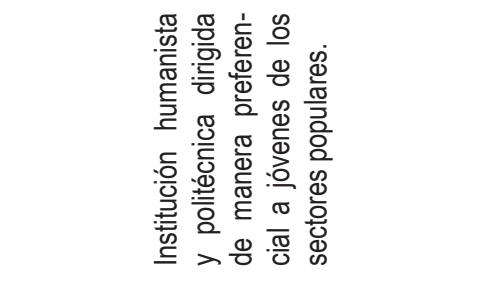 & 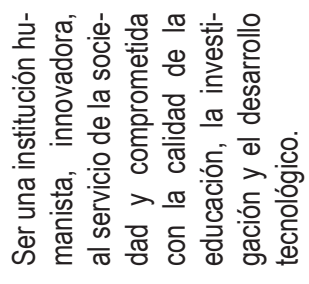 \\
\hline$\infty$ & 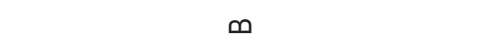 & $\infty$ \\
\hline 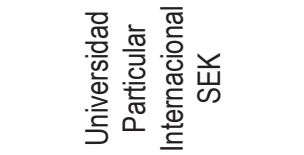 & 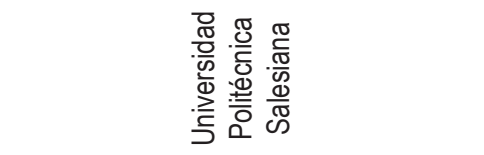 & 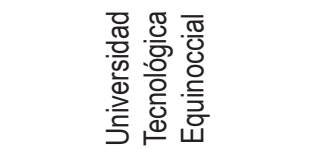 \\
\hline
\end{tabular}




\begin{tabular}{|c|c|c|c|c|}
\hline 孞 & $\frac{\frac{\pi}{0}}{\frac{0}{2}}$ & 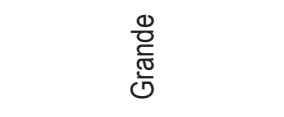 & $\begin{array}{l}\frac{\pi}{0} \\
\frac{\pi}{\pi} \\
0\end{array}$ & $\frac{\frac{\pi}{0}}{\frac{\pi}{2}}$ \\
\hline 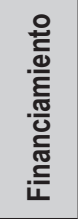 & 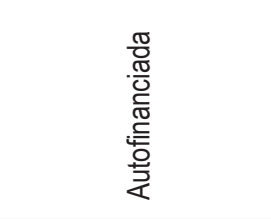 & 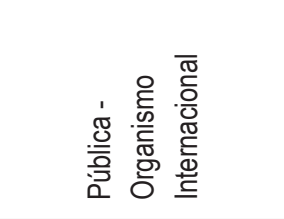 & 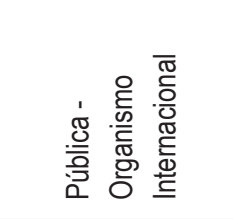 & $\begin{array}{l}\frac{.0}{0} \\
\frac{0}{2} \\
0\end{array}$ \\
\hline 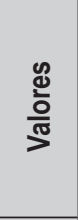 & 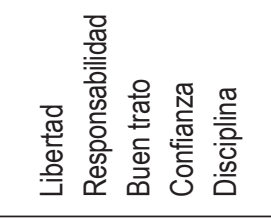 & 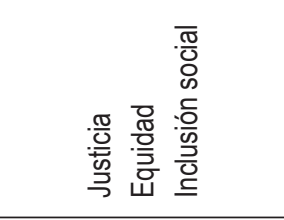 & & \\
\hline 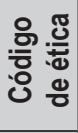 & $\bar{\omega}$ & $\bar{\omega}$ & 은 & 울 \\
\hline$\stackrel{\frac{0}{0}}{\frac{0}{5}}$ & 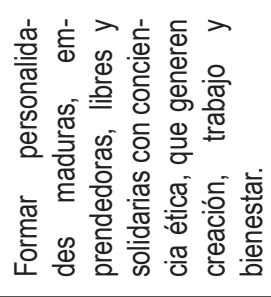 & 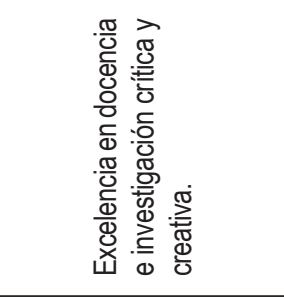 & 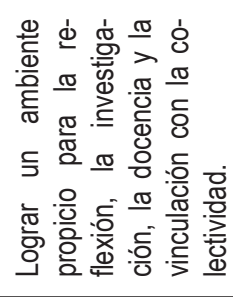 & 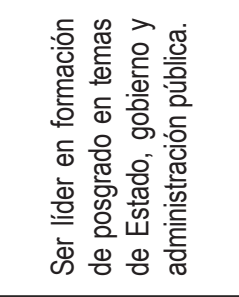 \\
\hline$\frac{\overline{0}}{\frac{0}{n}}$ & 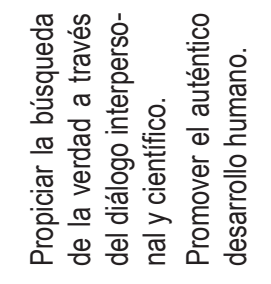 & 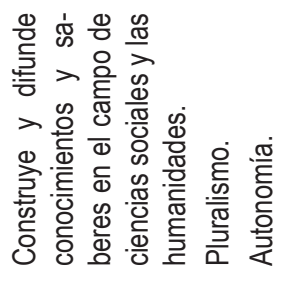 & 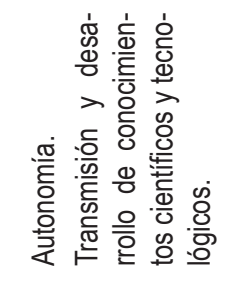 & 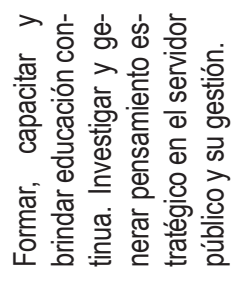 \\
\hline 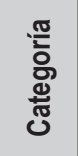 & $\infty$ & $\ll$ & $\varangle$ & $\infty$ \\
\hline 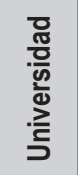 & 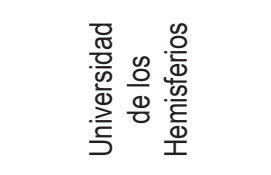 & 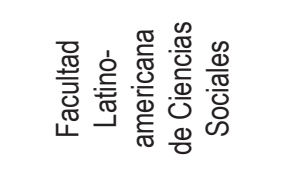 & 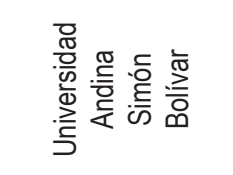 & 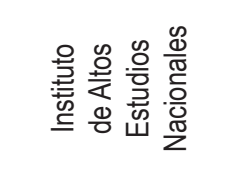 \\
\hline
\end{tabular}




\section{Entrevistas a profundidad}

En el presente trabajo se utilizó la entrevista como instrumento de investigación, lo que permitió recoger información sobre cómo actúan, piensan y sienten los gestores de talento humano.

\section{Agrupación de la información e identificación de variables}

Para recopilar la información o los datos se utilizó una batería de 33 preguntas subdivididas en tres variables:

- Ética profesional

- Estructura motivacional

- Autorregulación

\section{Variable 1: ética profesional}

- Liderazgo

- Ética, moral y valores

- Códigos conductuales

- Ética profesional e institucional

\section{Variable 2: estructura motivacional}

- Motivaciones personales

- Factores motivacionales

\section{Variable 3: autorregulación}

Cómo asume el gestor de talento humano en el ejercicio de su función:

- La comunicación

- El conflicto

- El mando o poder

- Las relaciones humanas 


\section{Resultados de las entrevistas}

A continuación se puede apreciar, en la figura 1, una representación en nube del estudio de contenido realizado con el software NVivo, en la que se muestran las palabras más frecuentes en las entrevistas realizadas.

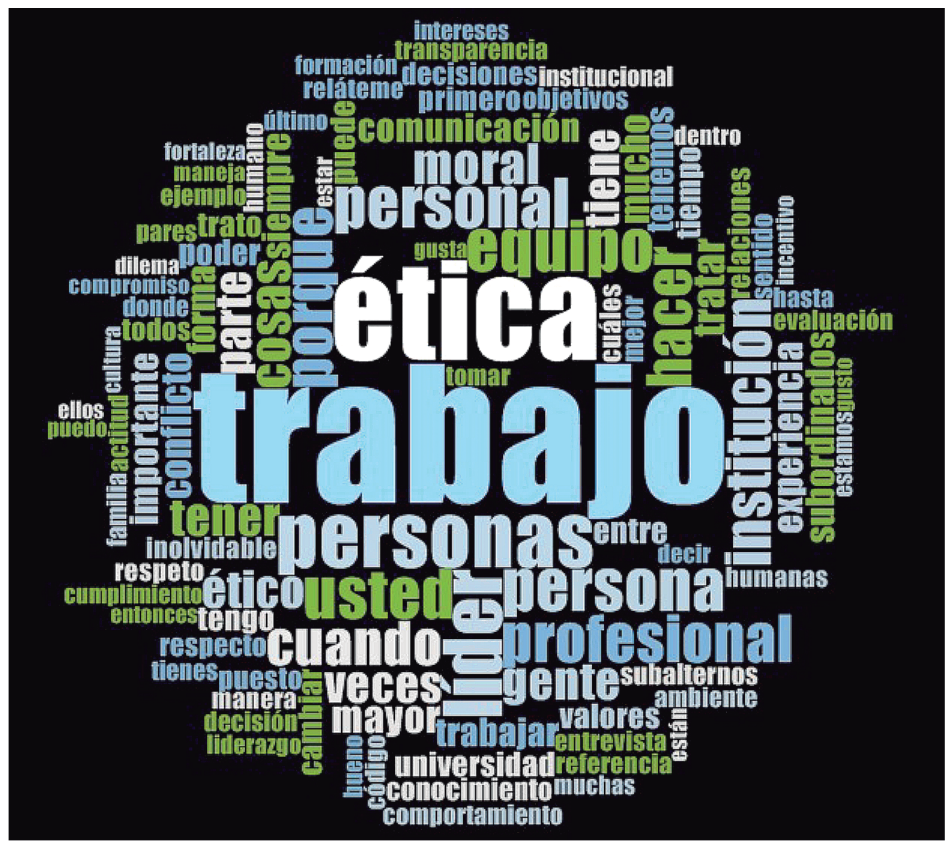

Las universidades públicas se gobiernan con base en la Ley Orgánica de Educación Superior (EC 2019) y en la normativa legal correspondiente, y cumplen con procesos basados en los reglamentos de la LOES y la Constitución. Por su parte, las universidades de carácter privado pueden flexibilizar un poco más sus normativas o procesos.

El software que se utilizó para la presente investigación trabaja con nodos, recipientes para poner las ideas de un proyecto determinado. Para el análisis de datos y para esta investigación, los nodos son los datos generales -es decir, las preguntas-, llamados categorías, y los subnodos son las respuestas a esas preguntas, llamadas subcategorías. 


\section{Tablas de resultados}

A continuación, se muestra de manera condensada el análisis cualitativo de datos realizado con NVivo, que permite categorizar por frecuencia e importancia:

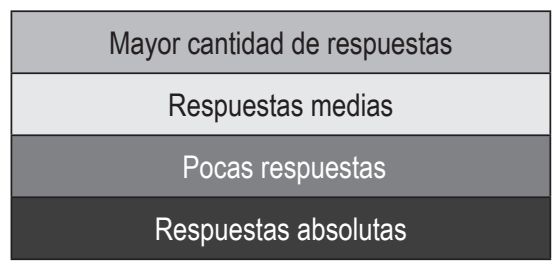

Hay que tener en cuenta que las respuestas que no están subrayadas fueron enunciadas, pero no fueron tendencia.

En la tabla 3 se observan las preguntas y las respuestas a ellas. El color marca la tendencia y las relaciones cualitativas se describen al final.

Tabla 3

\section{Resultados de la entrevista "Ética profesional"}

\begin{tabular}{|c|c|c|}
\hline \multirow{3}{*}{1.} & \multirow{3}{*}{ ¿Cómo usted se define como líder? } & Líder situacional. \\
\hline & & Líder burocrático. \\
\hline & & Líder democrático. \\
\hline \multirow{6}{*}{2.} & \multirow{6}{*}{ ¿Qué son para usted la ética y la moral? } & Los valores son esquemas mentales. \\
\hline & & $\begin{array}{l}\text { La moral es un código de conducta que regu- } \\
\text { la el comportamiento. }\end{array}$ \\
\hline & & La moral está relacionada con los valores. \\
\hline & & $\begin{array}{l}\text { La ética y la moral están orientadas a la leal- } \\
\text { tad institucional. }\end{array}$ \\
\hline & & La ética y la moral son constructos culturales. \\
\hline & & $\begin{array}{l}\text { La ética tiene bases muy profundas en el ser } \\
\text { humano. }\end{array}$ \\
\hline
\end{tabular}




\begin{tabular}{|c|c|c|}
\hline \multirow{8}{*}{3.} & \multirow{8}{*}{ ¿Cómo aplica en su vida personal la ética? } & Rectitud de procedimientos. \\
\hline & & No solo pareciendo sino siendo. \\
\hline & & El límite de la ética es cuando daño a alguien. \\
\hline & & Inculcando valores. \\
\hline & & Cumpliendo perfectamente mis obligaciones. \\
\hline & & Aplicando la normativa sin distinciones. \\
\hline & & $\begin{array}{l}\text { Con un comportamiento idóneo para mante- } \\
\text { ner un buen clima laboral. }\end{array}$ \\
\hline & & Aplicándolo en la realidad o cotidianidad. \\
\hline \multirow{2}{*}{4.} & \multirow{2}{*}{$\begin{array}{l}\text { ¿Cómo usted lleva su compromiso de trans- } \\
\text { parencia en su trabajo? }\end{array}$} & Comportamiento actitudinal. \\
\hline & & Aplicación a la normativa. \\
\hline \multirow{3}{*}{5.} & \multirow{3}{*}{$\begin{array}{l}\text { ¿Cuáles son las prácticas comunes de ética y } \\
\text { moral que realiza en su trabajo? }\end{array}$} & Velar y cuidar a las personas. \\
\hline & & Respeto a la norma. \\
\hline & & Empoderamiento. \\
\hline \multirow{3}{*}{6.} & \multirow{3}{*}{$\begin{array}{l}\text { Cuando uno de subalternos infringe el código } \\
\text { de conducta de la institución, ¿qué es lo pri- } \\
\text { mero que usted hace? }\end{array}$} & Procedo según la norma. \\
\hline & & Confrontación de actores. \\
\hline & & Actitud reflexiva. \\
\hline \multirow{6}{*}{7.} & \multirow{6}{*}{$\begin{array}{l}\text { ¿Qué prácticas referentes a la ética profesio- } \\
\text { nal desearía cambiar en sus subordinados? }\end{array}$} & El trabajo en equipo. \\
\hline & & Los privilegios. \\
\hline & & No individualizar. \\
\hline & & Integración del equipo de trabajo. \\
\hline & & Ética y actitud. \\
\hline & & Comunicación. \\
\hline
\end{tabular}


Ethos en el liderazgo de los gestores de talento humano de las universidades y escuelas politécnicas

\begin{tabular}{|c|c|c|}
\hline \multirow{9}{*}{8.} & \multirow{9}{*}{$\begin{array}{l}\text { ¿Cuáles son los valores esenciales que rigen } \\
\text { su diario vivir? }\end{array}$} & Transparencia. \\
\hline & & Tolerancia. \\
\hline & & Responsabilidad. \\
\hline & & Respeto. \\
\hline & & Lealtad. \\
\hline & & Humildad. \\
\hline & & Honestidad. \\
\hline & & Comportamiento. \\
\hline & & Amor. \\
\hline \multirow{4}{*}{9.} & \multirow{4}{*}{$\begin{array}{l}\text { ¿Qué mecanismos utiliza usted para evaluar } \\
\text { periódicamente a sus subalternos, además de } \\
\text { la evaluación de desempeño de la institución? }\end{array}$} & Evaluación por feedback. \\
\hline & & Evaluación de subalterno. \\
\hline & & Evaluación $360^{\circ}$. \\
\hline & & Autoevaluación. \\
\hline \multirow{4}{*}{10.} & \multirow{4}{*}{$\begin{array}{l}\text { ¿Cómo evita el conflicto de intereses entre } \\
\text { sus subordinados y el personal de la institu- } \\
\text { ción? }\end{array}$} & Siendo justo y equitativo. \\
\hline & & Escucha activa. \\
\hline & & Comunicación activa y oportuna. \\
\hline & & Aplicando normativa. \\
\hline \multirow{6}{*}{11.} & \multirow{6}{*}{$\begin{array}{l}\text { Cuando se encuentra en un dilema ético, ¿en } \\
\text { que se apoya para solucionarlo, profesional y } \\
\text { personalmente? }\end{array}$} & Reflexión de pros y contras. \\
\hline & & Norma. \\
\hline & & Conocimientos que poseo. \\
\hline & & Comunicación activa. \\
\hline & & Busco apoyo y consejo. \\
\hline & & Ética, valores y principios. \\
\hline
\end{tabular}




\begin{tabular}{|c|c|c|}
\hline \multirow{5}{*}{12.} & \multirow{5}{*}{$\begin{array}{l}\text { Reláteme una experiencia inolvidable en refe- } \\
\text { rencia a un evento ético }\end{array}$} & Promociones 0 ascenso \\
\hline & & Mantener la ética. \\
\hline & & Inolvidables positivas. \\
\hline & & Inolvidables negativas. \\
\hline & & Ayudar sin interés. \\
\hline \multirow{7}{*}{13.} & \multirow{7}{*}{ ¿Cuál es su mayor fortaleza como líder? } & Orientación al logro. \\
\hline & & Influencia. \\
\hline & & Experiencia. \\
\hline & & Ética y valores. \\
\hline & & Desarrollo de personas. \\
\hline & & Carácter. \\
\hline & & Adaptación al cambio. \\
\hline \multirow{14}{*}{14.} & \multirow{14}{*}{$\begin{array}{l}\text { ¿Cuál es su mayor cualidad como ser huma- } \\
\text { no? }\end{array}$} & Solidario. \\
\hline & & Humano. \\
\hline & & Sensible. \\
\hline & & Respetuoso. \\
\hline & & Minucioso. \\
\hline & & Humilde. \\
\hline & & Honesto. \\
\hline & & Generoso. \\
\hline & & Fiable. \\
\hline & & Explorador, ingenioso. \\
\hline & & Escucha proactiva. \\
\hline & & Empatía. \\
\hline & & Confiable. \\
\hline & & Adaptable. \\
\hline
\end{tabular}


Ethos en el liderazgo de los gestores de talento humano de las universidades y escuelas politécnicas

\begin{tabular}{|c|c|c|}
\hline \multirow{7}{*}{15.} & \multirow{7}{*}{ ¿Cómo toma usted las decisiones difíciles? } & Siendo justo. \\
\hline & & Reuniones de trabajo. \\
\hline & & Apegado a la norma. \\
\hline & & Apegado a la lógica. \\
\hline & & Cabeza fría. \\
\hline & & Apoyo en consejos. \\
\hline & & Análisis profundo. \\
\hline \multirow{5}{*}{16.} & \multirow{5}{*}{$\begin{array}{l}\text { ¿La formación de la universidad contribuyó a } \\
\text { su rol de líder con respecto al conocimiento, } \\
\text { actitud, comportamiento, otros...? }\end{array}$} & Sí. \\
\hline & & No. \\
\hline & & El líder se hace. \\
\hline & & Conocimientos y experiencias. \\
\hline & & Aprendizaje constante. \\
\hline \multirow{9}{*}{17.} & \multirow{9}{*}{ ¿Cuál es su mayor debilidad como líder? } & Ser poco flexible. \\
\hline & & Ser confiado. \\
\hline & & No incentivo a mi equipo. \\
\hline & & Muy sensible. \\
\hline & & Impaciencia. \\
\hline & & Imagen de seriedad. \\
\hline & & Frustración con el trabajo de equipo. \\
\hline & & Desconfiado. \\
\hline & & Hacer suposiciones. \\
\hline \multirow{5}{*}{18.} & \multirow{5}{*}{$\begin{array}{l}\text { ¿Cuál fue su último trabajo, cuál fue su posi- } \\
\text { ción y puesto? }\end{array}$} & Secretaria. \\
\hline & & Jefe administrativo. \\
\hline & & Director de Talento Humano. \\
\hline & & Coordinador de Talento Humano. \\
\hline & & Consultor de Talento Humano. \\
\hline
\end{tabular}




\begin{tabular}{|c|c|c|}
\hline 19. & $\begin{array}{l}\text { ¿Usted cree que, por orden de un superior, } \\
\text { podría cambiar su pensamiento respecto a la } \\
\text { ética y la moral? }\end{array}$ & No. \\
\hline \multirow{5}{*}{20.} & \multirow{5}{*}{$\begin{array}{l}\text { ¿Cómo logra un equilibrio entre su ética pro- } \\
\text { fesional y la ética institucional? }\end{array}$} & Son equilibrados. \\
\hline & & No hay equilibrio. \\
\hline & & Ejercicios mentales y análisis. \\
\hline & & Basándose en la norma. \\
\hline & & Antecediendo en beneficio de la institución. \\
\hline \multirow{6}{*}{21.} & \multirow{6}{*}{$\begin{array}{l}\text { Reláteme una experiencia inolvidable en refe- } \\
\text { rencia al liderazgo. }\end{array}$} & Ser éticos. \\
\hline & & $\begin{array}{l}\text { Realización de trabajos en selección de per- } \\
\text { sona. }\end{array}$ \\
\hline & & No doble moral. \\
\hline & & Motivación e incentivo. \\
\hline & & Conocimiento de cultura. \\
\hline & & Bienestar de grupo. \\
\hline
\end{tabular}

Fuente y elaboración propia.

En lo referente a la ética profesional, la entrevista corrobora que el recurso humano de las instituciones educativas es el capital más importante para los gestores, pues se entiende que el éxito o fracaso de las universidades o escuelas politécnicas depende del desempeño laboral de las personas que pertenecen a ellas. La mayoría de gestores se definen como líderes burocráticos, es decir, sus acciones están marcadas según la hoja de ruta de la institución y siguen al pie de la letra las reglas y la normativa.

Es por las funciones que realizan en las instituciones educativas que los gestores de talento humano deben tener bases muy profundas en la ética y en los valores personales (considerados esquemas mentales). Las tareas a realizar en su departamento son de vital importancia; entre las más sobresalientes encontramos: seleccionar a la persona idónea para un puesto determinado, evitar la alta rotación del personal y el personal insatisfecho, lograr el com- 
promiso de su equipo de trabajo, y articular un plan de carrera ético y sin favoritismo de ninguna especie.

El gestor de talento humano es el encargado de alinear el área de Talento Humano con los objetivos de la institución; por este motivo, al realizar sus obligaciones inculcan valores y aplican la normativa sin distinción.

Las estrategias en la dirección del talento humano se caracterizan por el cumplimiento de funciones repetitivas en los diferentes subsistemas. Las prácticas más comunes reconocidas por los gestores de talento humano son las de respeto a la norma, velar y cuidar de las personas, y empoderamiento. Las prácticas que quisieran cambiar en sus subordinados son las de ética, actitud e integración del trabajo en equipo.

Al hablar sobre ética profesional se indagó sobre los valores esenciales que rigen su cotidianidad, y se obtuvo que los valores más destacados, en la gran mayoría de gestores, son la transparencia, la responsabilidad, el respeto, la honestidad y, como complemento a lo expuesto anteriormente, tratar de ser justos y equitativos en todos sus actos.

Consideran también que sus mayores fortalezas, como líderes, son la influencia, la experiencia y el carácter. Por otro lado, perciben que sus mayores debilidades son hacer suposiciones acerca del trabajo de las personas de su equipo, ser muy sensibles y desconfiados, debido a la consciente influencia que tienen con sus subalternos.

Los gestores, en su diario vivir, no solo tienen fortalezas, sino que también gozan de cualidades humanas: la solidaridad, la humildad, la empatía y la sensibilidad. Al igual que el resto de profesionales, también tienen que tomar decisiones difíciles, y entonces recurren a un análisis profundo, con cabeza fría; sin embargo, tienen sumamente claro que sus decisiones afectan vidas y familias.

Ninguno de los entrevistados cambiaría su pensamiento respecto a la ética y la moral. La mayoría está alineado a la ética empresarial y lucha constantemente para que todos sus actos contribuyan al crecimiento institucional, guiando y procurando siempre el bienestar de grupo, tratando de llegar a sus subalternos con motivación emocional, para que se empoderen de su puesto y de las funciones atribuidas a él. 
Tabla 4

Resultados de la entrevista "Estructura motivacional"

\begin{tabular}{|c|c|c|}
\hline \multirow{4}{*}{1.} & \multirow{4}{*}{ ¿Qué es lo que lo motiva a trabajar? } & Reconocimiento. \\
\hline & & Ganar experiencia y continuar aprendiendo. \\
\hline & & Familia. \\
\hline & & El poder servir. \\
\hline \multirow{2}{*}{2.} & \multirow{2}{*}{ ¿Qué es lo más importante en su vida? } & Yo. \\
\hline & & Familia. \\
\hline \multirow{3}{*}{3.} & \multirow{3}{*}{$\begin{array}{l}\text { ¿Se ha sentido obligado a ir a su lugar de } \\
\text { trabajo? }\end{array}$} & Nunca. \\
\hline & & Pereza. \\
\hline & & En ciertas ocasiones sí. \\
\hline \multirow{4}{*}{4.} & \multirow{4}{*}{$\begin{array}{l}\text { ¿Qué desearía tener en su trabajo para sen- } \\
\text { tirse más a gusto? }\end{array}$} & Privacidad. \\
\hline & & Personalizar la oficina. \\
\hline & & Mayor autonomía. \\
\hline & & Equipo de trabajo fiable. \\
\hline
\end{tabular}

Fuente y elaboración propia.

El análisis motivacional de los gestores de talento humano da una idea clara acerca de sus motivaciones intrínsecas y extrínsecas. Cuando se les pregunta sobre qué los motiva a trabajar, contestan: la experiencia, continuar aprendiendo, poder servir y el reconocimiento.

Un lugar importante en su vida lo ocupa su familia, y están altamente motivados porque les gusta lo que hacen. Sobresale en las respuestas que su mayor anhelo no es el económico, sino poder personalizar su oficina y obtener mayor autonomía en su labor. 
Ethos en el liderazgo de los gestores de talento humano de las universidades y escuelas politécnicas

\section{Tabla 5}

Resultados de la entrevista "Autorregulación"

\begin{tabular}{|c|c|c|}
\hline \multicolumn{3}{|c|}{ ¿Cómo maneja usted en el ejercicio de su profesión? } \\
\hline \multirow{7}{*}{1.} & \multirow{7}{*}{ El conflicto } & Siendo objetivo. \\
\hline & & Siendo mediador. \\
\hline & & Mediante la norma. \\
\hline & & Evitando juzgar. \\
\hline & & Escuchando a las partes. \\
\hline & & Buscando soluciones. \\
\hline & & Buscando información. \\
\hline \multirow{9}{*}{2.} & \multirow{9}{*}{ El mando o poder } & Sin abuso. \\
\hline & & Para lograr influencia y convencimiento. \\
\hline & & Para ayudar. \\
\hline & & Sin perder el equilibrio personal. \\
\hline & & Lo aplico con la norma. \\
\hline & & Con respeto hacia la otra persona. \\
\hline & & Con firmeza. \\
\hline & & Con ejemplos. \\
\hline & & Con ejercicios de humildad. \\
\hline \multirow{4}{*}{3.} & \multirow{4}{*}{ Las relaciones humanas con subordinados } & Son buenas. \\
\hline & & Equilibrio entre exigencia y mente abierta. \\
\hline & & Con base en el reconocimiento. \\
\hline & & Con respeto. \\
\hline
\end{tabular}




\begin{tabular}{|c|c|c|}
\hline \multirow{7}{*}{4.} & \multirow{7}{*}{ Las relaciones humanas con pares } & Son buenas. \\
\hline & & Con política. \\
\hline & & Serias y de cumplimiento. \\
\hline & & No son buenas. \\
\hline & & Llevaderas. \\
\hline & & Con respeto. \\
\hline & & Celo profesional \\
\hline \multirow{4}{*}{5.} & \multirow{4}{*}{ El cumplimiento de objetivos } & Planificación y evaluación. \\
\hline & & Estricto. \\
\hline & & Control y seguimiento. \\
\hline & & Con mucho trabajo. \\
\hline \multirow{6}{*}{6.} & \multirow{6}{*}{ La satisfacción en el ambiente de trabajo } & Motivación. \\
\hline & & Empatía. \\
\hline & & Comunicación. \\
\hline & & Comprensión. \\
\hline & & Buenas condiciones de trabajo. \\
\hline & & Buen trato. \\
\hline \multirow{5}{*}{7.} & \multirow{5}{*}{ El incentivo } & Sentido de pertenencia. \\
\hline & & Por cumplimento. \\
\hline & & No hay incentivo. \\
\hline & & No económicos, sino morales. \\
\hline & & Ambiente familiar. \\
\hline \multirow{4}{*}{8.} & \multirow{4}{*}{ La comunicación efectiva } & Sí se logra. \\
\hline & & Difícil de mantener. \\
\hline & & Con feedback. \\
\hline & & Canales de comunicación. \\
\hline
\end{tabular}

Fuente y elaboración propia. 
Los gestores de talento humano, en lo referente al manejo del conflicto, el poder, las relaciones humanas con subordinados y pares, la satisfacción en el ambiente de trabajo, el incentivo y la comunicación efectiva, manifiestan que lo más difícil de manejar con los subalternos es la comunicación.

Están conscientes de que la satisfacción en el ambiente de trabajo se logra con buen trato, empatía y buena comunicación, y que los incentivos económicos de a poco se han ido perdiendo, pero es muy gratificante proporcionar incentivos morales. Recordemos también que el gestor no solamente administra personas, sino corazones y emociones.

Tabla 6

Perfil de gestor de talento humano

\begin{tabular}{|c|c|}
\hline Nivel & Directivo \\
\hline Unidad o proceso & Departamento de Recursos Humanos \\
\hline Instrucción formal & Tercer nivel \\
\hline Título requerido & $\begin{array}{l}\text { - Ingeniero en Administración / Recursos Humanos } \\
\text { - Doctor en Administración } \\
\text { - Licenciado en Administración } \\
\text { - Psicólogo Industrial } \\
\text { - Carreras afines a Recursos Humanos } \\
\end{array}$ \\
\hline Misión & $\begin{array}{l}\text { Administrar, planificar, dirigir y controlar la ejecución de las actividades del depar- } \\
\text { tamento de Talento Humano. }\end{array}$ \\
\hline $\begin{array}{l}\text { Conocimientos } \\
\text { específicos }\end{array}$ & $\begin{array}{l}\text { - Control y evaluación de procesos de desarrollo institucional y de recursos humanos } \\
\text { - Planificación estratégica de recursos humanos, servicios y procesos para los } \\
\text { clientes organizacionales } \\
\text { - Normativa legal vigente en materia de talento humano } \\
\text { - Manejo sistemático de la gestión organizacional } \\
\text { - Análisis interpretativo de la información } \\
\text { - pormulación de planes operativos y de contingencia a corto, mediano y largo } \\
\text { - Planificación operativa de la gestión interna del departamento de Recursos Hu- } \\
\text { manos }\end{array}$ \\
\hline $\begin{array}{l}\text { Destrezas } \\
\text { o habilidades }\end{array}$ & $\begin{array}{l}\text { - Desarrollo estratégico de recursos humanos } \\
\text { - Orientación al logro } \\
\text { - Pensamiento analítico } \\
\text { - Pensamiento estratégico } \\
\text { - Pensamiento conceptual } \\
\text { - Planificación y gestión } \\
\text { - Iniciativa }\end{array}$ \\
\hline
\end{tabular}




\begin{tabular}{|c|c|c|}
\hline Experiencia & \multicolumn{2}{|l|}{3 años o más } \\
\hline $\begin{array}{l}\text { Conocimientos } \\
\text { complementarios }\end{array}$ & \multicolumn{2}{|c|}{$\begin{array}{l}\text { - Diseño de procesos y estructuras organizacionales } \\
\text { - Gestión de recursos humanos por competencias } \\
\text { - Manejo de recursos organizacionales } \\
\text { - Dirección y control de procesos de recursos humanos }\end{array}$} \\
\hline $\begin{array}{l}\text { Actividades } \\
\text { esenciales }\end{array}$ & \multicolumn{2}{|c|}{$\begin{array}{l}\text { - Dirige y evalúa la gestión del departamento de Talento Humano por procesos. } \\
\text { - Elabora planes de mejoramiento referentes a la administración del talento hu- } \\
\text { mano. } \\
\text { - Suscribe informes técnicos legales para movimientos de personal. } \\
\text { - Reformula la estrategia para el manejo del modelo de talento humano institucional. } \\
\text { - Elabora y reformula instrumentos, normas y disposiciones legales apegadas al } \\
\text { código de trabajo. }\end{array}$} \\
\hline \multirow{5}{*}{$\begin{array}{l}\text { Eje de competen- } \\
\text { cias sociales }\end{array}$} & $\begin{array}{l}\text { Competencias } \\
\text { personales transferibles }\end{array}$ & $\begin{array}{l}\text { - Habilidad de comunicación } \\
\text { - Trabajo en equipo } \\
\text { - Orientación al logro } \\
\text { - Toma de decisiones } \\
\text { - Capacidad negociadora } \\
\text { - Aprendizaje continuo } \\
\text { - Escucha efectiva }\end{array}$ \\
\hline & $\begin{array}{l}\text { Competencias } \\
\text { técnicas o profesionales }\end{array}$ & $\begin{array}{l}\text { - Manejo de prácticas de talento humano (atracción y } \\
\text { retención) } \\
\text { - Gestión cultural }\end{array}$ \\
\hline & $\begin{array}{l}\text { Competencias } \\
\text { de gestión }\end{array}$ & $\begin{array}{l}\text { - Pensamiento estratégico } \\
\text { - Gestión de procesos } \\
\text { - Orientación al cliente } \\
\text { - Gestión del cambio } \\
\text { - Liderazgo: personal con ciertas habilidades } \\
\text { - Interpersonarse a sí mismo, motivación } \\
\text { - trabajo en equipo, empatía }\end{array}$ \\
\hline & Competencias sociales & $\begin{array}{l}\text { - Empatía } \\
\text { - Sensibilidad social } \\
\text { - Capacidad de interrelación } \\
\text { - Facilitación } \\
\text { - Autocontrol }\end{array}$ \\
\hline & $\begin{array}{l}\text { Competencias } \\
\text { participativas o de for- } \\
\text { mas de organización }\end{array}$ & $\begin{array}{l}\text { - Poder de decisión con responsabilidad } \\
\text { - Dirección } \\
\text { - Coordinación } \\
\text { - Equidad } \\
\text { - Justicia }\end{array}$ \\
\hline
\end{tabular}




\begin{tabular}{|c|c|c|c|}
\hline $\begin{array}{l}\text { Valores } \\
\text { instrumentales }\end{array}$ & Ético-morales & $\begin{array}{l}\text { Cómo debería comportarse con } \\
\text { las personas a su alrededor }\end{array}$ & $\begin{array}{l}\text { - } \text { Honestidad } \\
\text { - Respeto } \\
\text { - Responsabilidad } \\
\text { - Lealtad } \\
\text { - Solidaridad } \\
\text { - Sinceridad } \\
\text { - Transparencia }\end{array}$ \\
\hline
\end{tabular}

Fuente y elaboración propia.

\section{Conclusiones}

El perfil de gestor de talento humano y la investigación realizada muestran que las habilidades duras (conocimiento, experiencia) de los entrevistados están comprobadas y no hay cuestionamientos, en vista de que su trayectoria y los años que llevan ejerciendo les permiten gestionar los subsistemas de su departamento de manera eficaz y eficiente.

En las entrevistas salió a relucir que hay que afianzar aspectos relacionados con el liderazgo, puesto que algunos de los entrevistados manifestaron que por las agobiadoras tareas que tienen a diario (orientación a la tarea) descuidan la parte primordial de las instituciones universitarias, que son sus subordinados. Hay que tener en cuenta que el liderazgo no solamente está orientado al cumplimiento de los objetivos institucionales, sino también a comprender, empoderar, desarrollar y motivar a las personas.

Se pudo evidenciar que las personas que ejercen la función de gestores de las universidades estudiadas gozan de confianza y estima por parte de las autoridades de las diversas instituciones, tienen experiencia probada de 3 a 25 años en cargos similares, se ajustan a la norma, y tratan de ser equilibradas, comprensivas y justas. 


\section{Referencias}

Aranguren, José Luis. 1958. Ética. Madrid: Revista de Occidente.

De Lella, Cayetano. 1999. "Modelos y tendencias de la formación docente". Conferencia del I Seminario Taller sobre el Perfil del Docente y Estrategias de Formación, Lima. https://bit.ly/38TJ4TV

EC CEAACES. 2015. Informe general sobre la evaluación, acreditación y categorización de las Universidades y Escuelas Politécnicas. https:/universidadsociedadec.files.wordpress.com/2014/04/ceaaces-informe-general-eval-accred-categn.pdf

EC. 2008. Constitución de la República del Ecuador. Registro Oficial 449, 20 de octubre.

Habermas, Jürgen. 1973. Problemas de legitimación en el capitalismo tardío. Madrid: Editorial Suhrkamp.

López Ruiz, Oswaldo. 2007. Ethos empresarial: el "capital humano” como valor social. Estudios Sociológicos. Ciudad de México: El Colegio de México.

Martínez, Horacio. 2010a. Liderazgo responsable. Bogotá: Ecoe Ediciones.

---. 2010b. Responsabilidad social y ética empresarial. Bogotá: Ecoe Ediciones.

Octavi, Fullat, y Clara Gomis. 1987. El hombre, un animal ético. Barcelona: Vicens-Vives.

Olaz, Angel. 2012. Manual de recursos humanos. Murcia: Servicio Universidad de Murcia.

Randall, Schuler, y Susan Jackson. 1987. "Linking Competitive Strategies with Human Resource Management Practices”. The Academy of Management Executive (42): 207-219. https://journals.aom.org/doi/abs/10.5465/ame.1987.4275740?journalCode=amp

Rodríguez Córdoba, María del Pilar, y Uriel Bustamante Lozano. 2008. "Desarrollo de competencias para el comportamiento ético-gerencial: un enfoque de responsabilidad". Cuadernos de Administración 21 (35): 205-228. https://bit.ly/3e8VYzQ

Romero Villagómez, Citlali, y María Teresa Yurén Camarena. 2007. "Ethos profesional, dispositivo universitario y conformación". Reencuentro (49), 22-29. http://www.redalyc. org/articulo.oa?id=34004904

Sancén Contreras, Fernando. 2013. "Ciencia y ethos: una ética para el futuro (a propósito de la Diskursethik de Jürgen Habermas)". Signos Filosóficos 15 (29): 39-69. https://bit. ly/2LRdQTF

Sonnenfeld, Alfred. 2012. Liderazgo ético: la sabiduría de decidir bien. Madrid: Encuentro.

Yuren, Teresa. 2005. "Ethos y autoformación en los dispositivos de formación de docentes". En Ethos y autoformación del docente. Análisis de dispositivos de formación de profesores, coordinado por Teresa Yurén, Cecilia Navia y Cony Saenger. Barcelona: Editorial Pomares. 